\title{
Education and Research at the Nexus of Food, Energy, and Water with a 3-Axis Farming Robot
}

\section{Dr. Abhijit Nagchaudhuri, University of Maryland, Eastern Shore}

Dr. Abhijit Nagchaudhuri is currently a Professor in the Department of Engineering and Aviation Sciences at University of Maryland Eastern Shore. He is a member American Society for Mechanical Engineers (ASME), American Society for Engineering Education (ASEE) and, American Society for Agricultural and Biological Engineers(ASABE) and is actively involved in teaching and research in the fields of (i) robotics and mechatronics, (ii)remote sensing and precision agriculture, and,(iii) biofuels and renewable energy. He has published more than 70 refereed articles in journals and conference proceedings. Dr. Nagchaudhuri received his baccalaureate degree from Jadavpur University in Kolkata, India with honors in Mechanical Engineering. Thereafter, he worked in a multinational industry for a little over three years before joining Tulane University as a graduate student in the fall of 1987. He received master's degree from Tulane University in 1989 and doctoral degree from Duke University 1992.

\section{Mr. Jesu Raj Pandya, University of Maryland Eastern Shore}

Currently a doctoral student in Food \& Agriculture Sciences, University of Maryland Eastern Shore (UMES), MSc - Applied Computer Sciences at UMES, BSc - Electrical and Electronical Engineering ( JNTU).

Interest in robotics and automation in food production and food safety.

\section{Dr. Madhumi Mitra Ph.D, University of Maryland Eastern Shore}

Dr. Madhumi Mitra is currently a professor of environmental sciences in the department of Natural Sciences at the University of Maryland Eastern Shore. She is also the coordinator of Biology and Chemistry Education. Dr. Mitra is actively involved in teaching and research in the areas of biofuels and renewable energy; applications of algae in food and environment; and water quality. She has published several peer-reviewed articles in journals and conference proceedings, and is the recipient of various awards and competitive grants. Dr. Mitra received her baccalaureate degree from Presidence College in Kolkata, India with honors in Plant Biology, Geology, and Physiology. She received her master's degree from University of Calcutta, India, and her doctoral degree from North Carolina State University, Raleigh, NC in 2002.

\section{Travis Ford, University of Maryland Eastern Shore}

Doctoral Student with a background in environmental science, agricultural science, and food science. 


\title{
Education and Research Platform at the Nexus of Food, Energy, and Water using a 3-Axis Farming Robot
}

\begin{abstract}
"Smart Farming" efforts at the University of Maryland Eastern Shore (UMES) have been incorporated with AIRSPACES (Autonomous Instrumented Robotic Sensory Platforms to Advance Creativity and Engage Students) project supported by Maryland Space Grant Consortium. Broad goals of the project are aligned with USDA's "environmentally friendly agriculture" and NASA's "earth science" mission objectives.

As part of the AIRSPACES project, the UMES team has developed and integrated autonomous platforms such as autonomous boats, autonomous ground robots, as well as unmanned aerial systems, to advance sustainable agricultural practices.

Farmbot, a small autonomous farming machine, has been recently acquired by the "Smart Farming" and "AIRSPACES" project team at UMES for conducting precision farming field experiments on a small scale. The machine seeds, kills weeds, senses soil moisture levels, and irrigates plants individually over 10 feet by 20 feet area. It is, in essence, a three-axis Cartesian robot quite similar to a 3D printer that runs on Raspberry Pi 3 and Arduino like microprocessor board. It can be easily manipulated using a web application over a smartphone. A borescope type camera (Pi-Cam) can be integrated with the z-axis of the machine that can be used for weed detection and time-lapse photography. Food Science and Technology graduate students are working in concert with engineering undergraduates and UMES staff to oversee the installation and to set up the preliminary experimental layout. Some of the pitfalls and successes during the installation of both the hardware and software of the mechatronic device will be highlighted in this paper. Initial plans of growing turnips with the FarmBot were unsuccessful due to the severity of winter weather. The project team has completed building a hoop house around the FarmBot to extend the growing season. Immediate installation plans for powering the FarmBot with solar panels and wind turbines and meeting its irrigation needs through rainwater harvesting are in progress.
\end{abstract}

\subsection{Introduction}

FarmBot efforts at the University of Maryland Eastern Shore(UMES) are integral to smart agriculture and precision farming efforts[1,2] funded by the National Institute of Food and Agriculture(NIFA) and Maryland Space Grant Consortium. Smart agriculture endeavors have been ongoing for several years now and have been instrumental in modernizing production agricultural practices undertaken by the farm personnel on campus. The broad multidisciplinary umbrella has also provided opportunities for a multidisciplinary team of undergraduate and graduate students to work in experiential and research projects involving automated yield monitoring using combine harvesters, sensor-based variable rate application, remote sensing using 
drones, water quality monitoring using robotic boats, deficit irrigation, subsurface drip irrigation, fertigation, and wireless soil moisture monitoring systems. Software tools for statistical analysis, geographical information systems(GIS), and, image processing, mosaicking, and mapping to document and analyze field experiments are also being utilized by the students in the project team.

The FarmBot efforts not only tie in well the overarching theme of the AIRSPACES project but provide a novel dimension to precision farming efforts on a smaller scale. Such small scale agricultural automation efforts are likely to work in concert with farm-to-table kitchens [3] that are growing in popularity in urban and suburban areas.

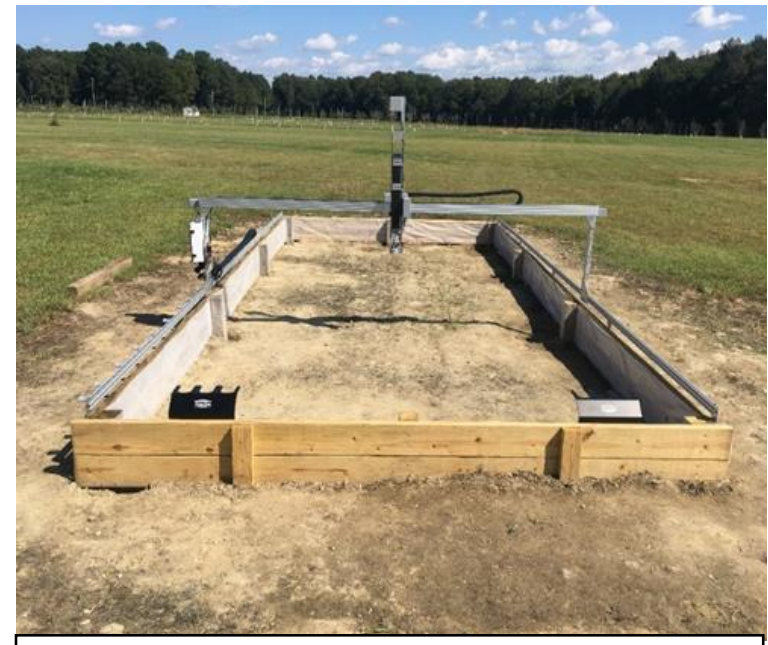

Figure 1: FarmBot and its' 10ft by $20 \mathrm{ft}$ bed

The FarmBot project is envisioned to be a demonstration platform for small scale sustainable food production [4] using a robotic device [5] powered by wind and solar energy[6] that utilizes harvested rainwater for irrigation. The FarmBot set up also provides a convenient platform to conduct small scale field experiments to investigate crop harvest characteristics based on a variety of inputs related to irrigation and nutrient levels. As mentioned earlier the FarmBot (Figure 1) has been installed at UMES to service a $10 \mathrm{ft}$ by $20 \mathrm{ft}$ bed. All the autonomous capabilities of the platform will be utilized and demonstrated in the preliminary study that is underway. The bed has been prepared with fertile soil. Varying amounts of hydrogel (recommended level $-30 \mathrm{~g} / 18 \mathrm{~kg}$ of soil, half of the recommended level and one and a half times the recommended level, and none ) were added to different sections of the raised bed to study the effects on the growth of root vegetables for an initial field trial. Hydrogels (Super Absorbent Polymers(SAP)) [7,8] are mostly used in sandy and semi-arid areas to increase arable lands to enhance agricultural production. They are referred to as hydrogel because of their water retaining properties and they tend to swell (Figure 2) by absorbing water from the soil when available. In dry conditions, the water is slowly released to the soil.

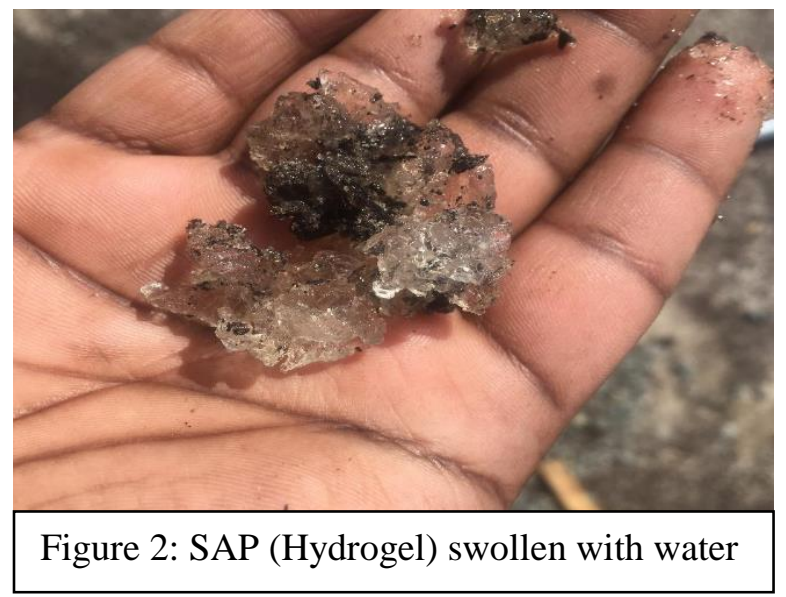

The first trial with growing turnip immediately after the basic set-up of the FarmBot was unsuccessful, largely due to the severity of weather that ensued after the seeds were planted. Subsequently, the project team has discarded the plans for using hydrogel and has decided to perform a similar field experiment in the 2020 spring that will study the effects of varying levels of irrigation applied using the FarmBot on selected leafy green and root vegetables. A small amount of urea has been added to the soil to raise the 
nitrogen level based on soil testing that was done after the failed trial with turnip.

The hoop house, wind turbine, and solar panel installations are almost complete at the time of writing this paper (Figure 3). The project team consisting of engineering and construction technology undergraduates, food science and technology graduate students, and university staff are in the process of finalizing the rainwater harvesting set up. In the meantime, however, the FarmBot can be operated with electrical connection and water supply pipes and fittings installed with the assistance of physical plant personnel at the university.

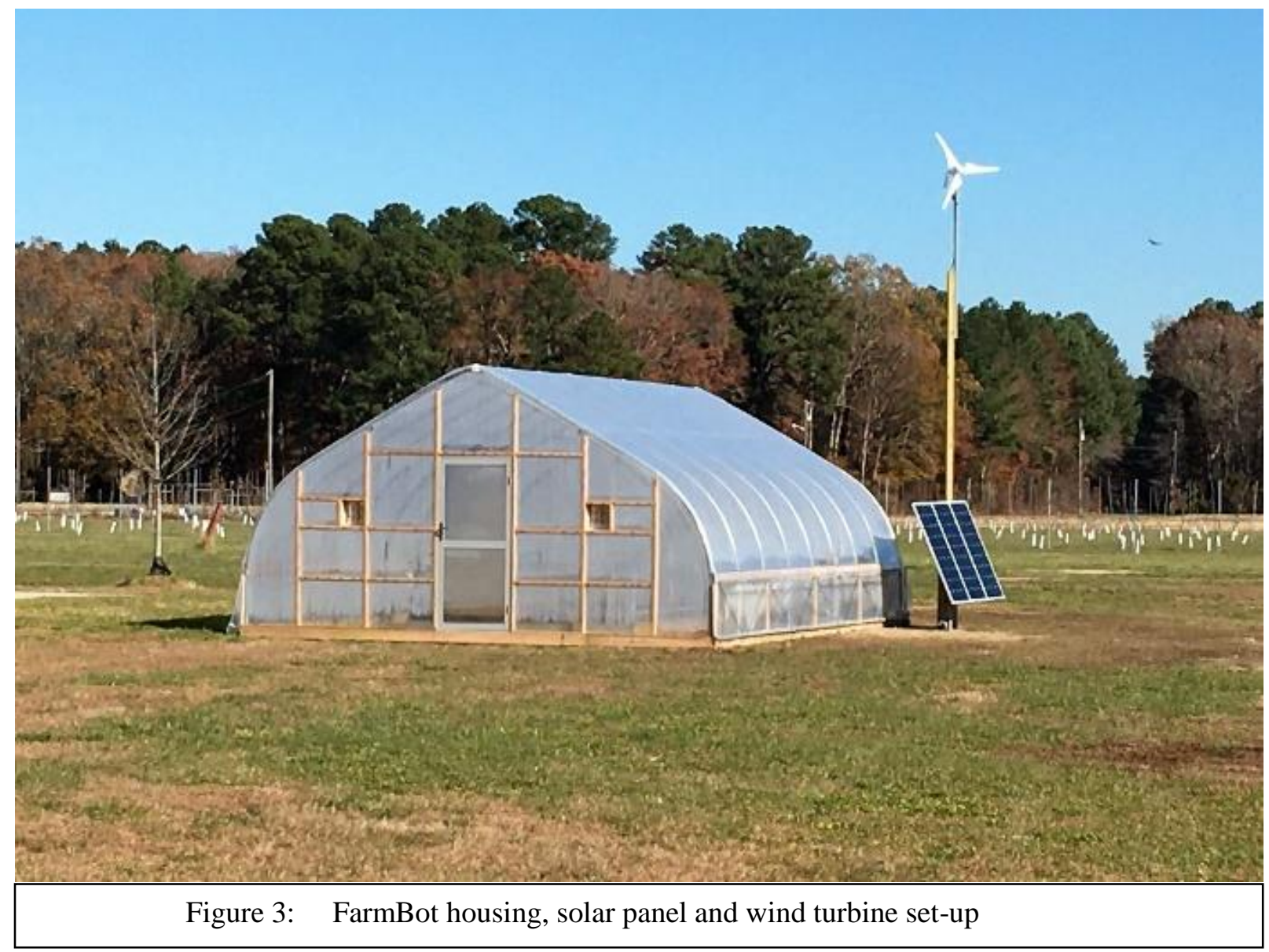

\subsection{FarmBot Genesis v1.4 XL at UMES}

FarmBot Genesis v1.4 XL is a 3-axis Cartesian robot. It is controlled by Raspberry Pi and Arduino microprocessor boards and uses encoders to command stepper motors for precision movement in all three axes. FarmBot Operating System (OS, FarmBot-rpi3-7.0.1) image file was downloaded and installed on an SD card using a software etcher and inserted in the SD card slot in the Raspberry Pi for boot up. On the first bootup, the FarmBot went through with the set-up with the configurator tool and enabled a Wi-Fi signal. The UMES Wi-Fi network has protective proximity. To overcome this problem, a TP-Link 300Mbps wireless router was used which enabled the team members of 


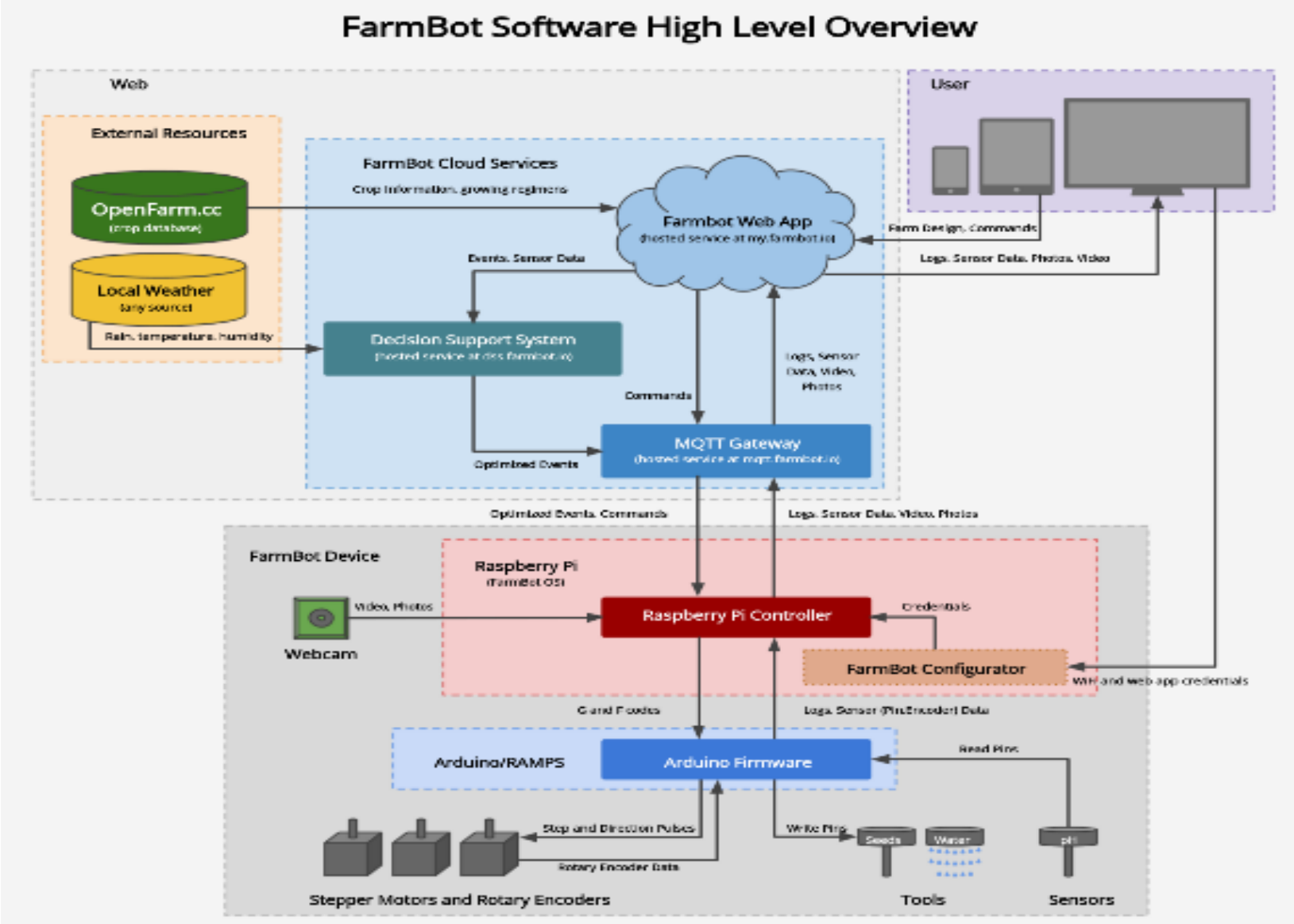

Figure 4: FarmBot Software- Overview

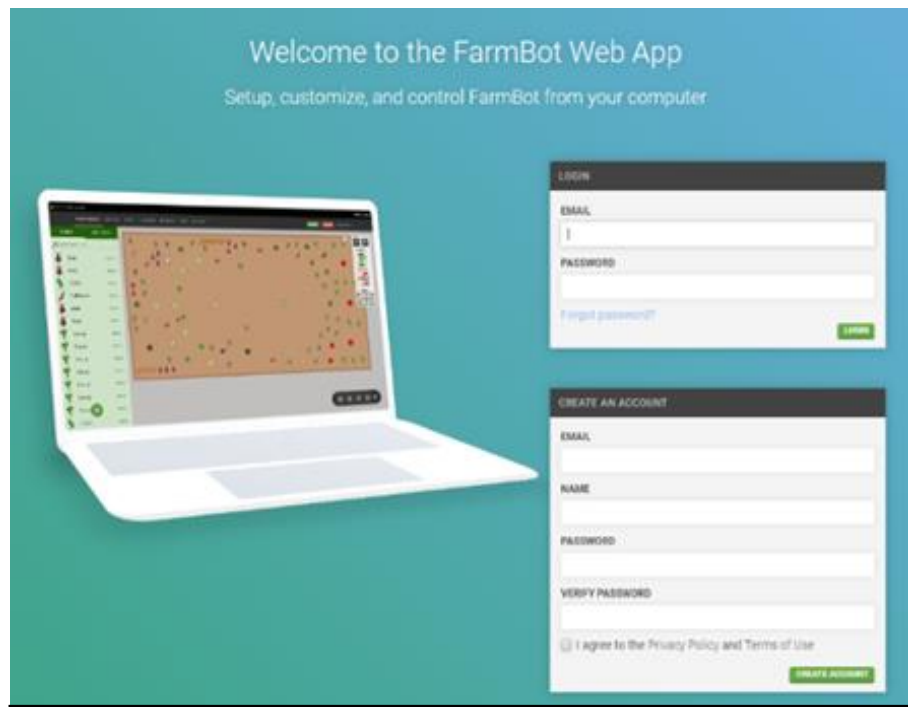

Figure 5: FarmBot Web App Login Page the FarmBot project to $\log$ in to the device. A Web App running on a personal cell-phone and/or laptop computer can be used to remotely operate the machine (see Figures 4 and 5).

The FarmBot kit comes with four tools (for seeding, weeding, watering, and soil moisture sensing), two toolbays, a seed bin, and two seed trays. The attachments for the seeder, weeder, watering nozzle tool and soil sensor, along with the seed bin and seed tray are shown in Figure 6. A Universal Tool Mount (UTM), that is mounted on the $\mathrm{z}$-axis of the robot allows the machine to easily switch tools to 


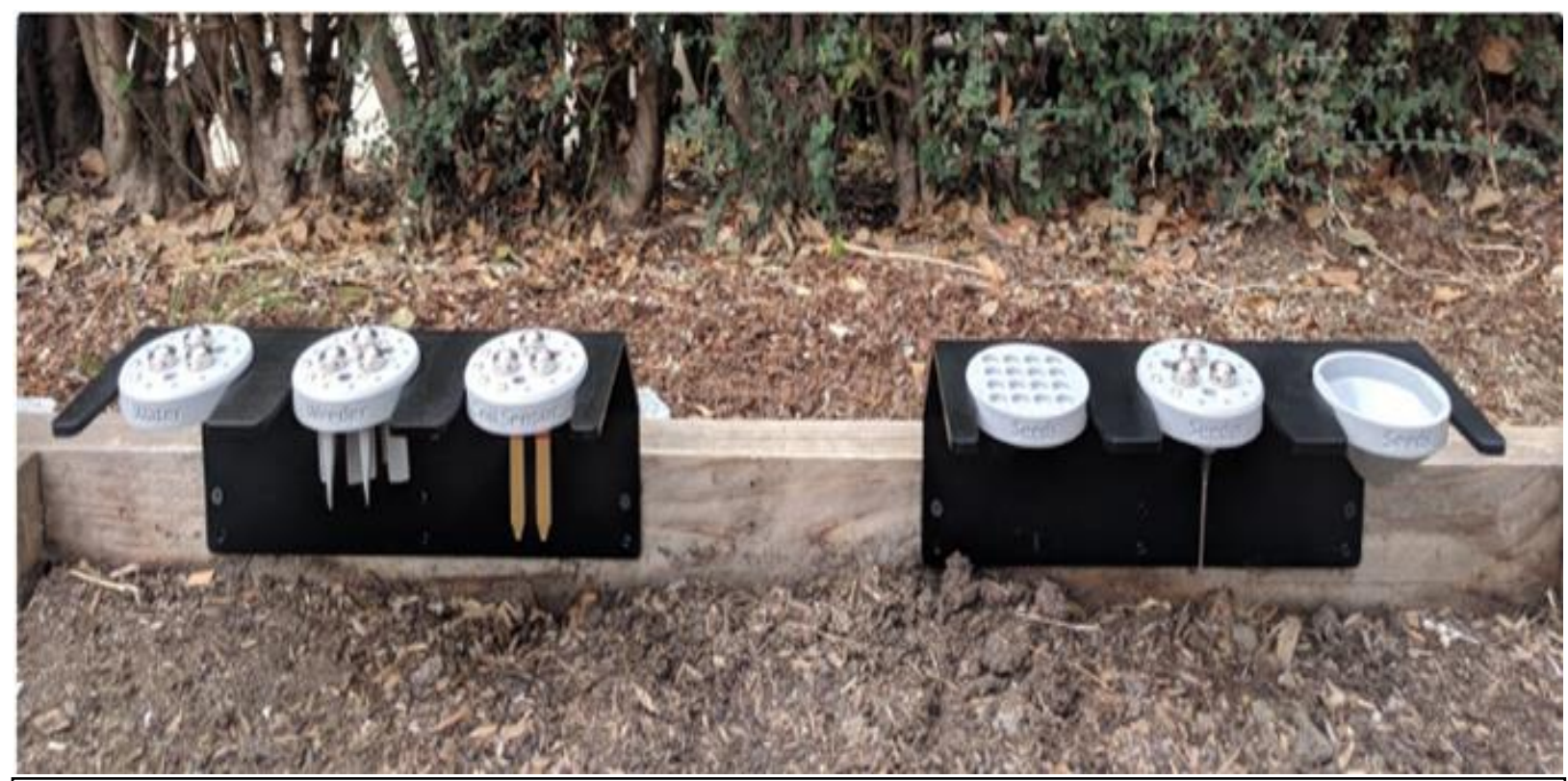

Figure 6: Watering Nozzles, Weeders, Soil Moisture Sensor, Seeder and two seed trays

execute different tasks. A camera has also been mounted next to the UTM on the z-axis of the FarmBot. It is a fully waterproof borescope style camera. The camera will be used to take photographs for weed detection and time-lapse photography of the plants as they grow.

\subsection{Pitfalls and Successes}

It took some time to complete the installation after the FarmBot kit arrived on campus in early summer (2019). Preparing the raised bed using wooden planks was time-consuming and the project team had to coordinate with the physical plant personnel at the university to get the water line and

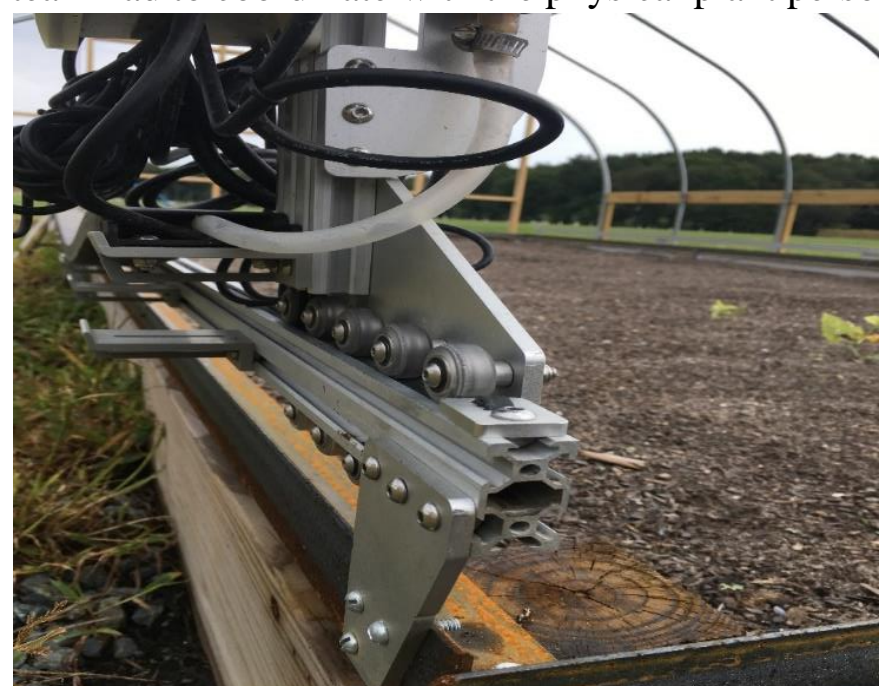

Figure 7: Farmbot $\mathrm{x}$-axis track on metal frame electricity hooked up to the FarmBot. The area (10ft by $20 \mathrm{ft}$ ) serviced by the machine was divided to accommodate four levels of hydrogel with two replicates. Enabling the WiFi set up for remote access to the FarmBot also presented some initial difficulties. By the time the initial difficulties were overcome and seeding was completed, it was late fall. The severity of the winter weather quickly followed and the field experiment planned did not succeed. However, the team learned valuable lessons concerning operational difficulties with the machine and logistics of conducting field experiments with it. 
A major issue that was not anticipated was the warping of the wood frame of the FarmBot bed which provided the stand for the motion along the X-axis (longitudinal) for the robot. This resulted in the FarmBot getting stuck without notice while performing automated task sequences. The project team aligned the $\mathrm{x}$-axis track on a metal frame as shown in Figure 7 to alleviate the problem. Sequencing trials performed subsequently have vastly improved the reliability of the device.

Some software limitations were observed that impeded operational efficiency. For instance, the absence of loop commands in the sequences made the input programming time-consuming. Also, the lack of a pause feature while running sequences made operational logistics complicated. The project team hopes to work with FarmBot software developers to address these software issues in the future.

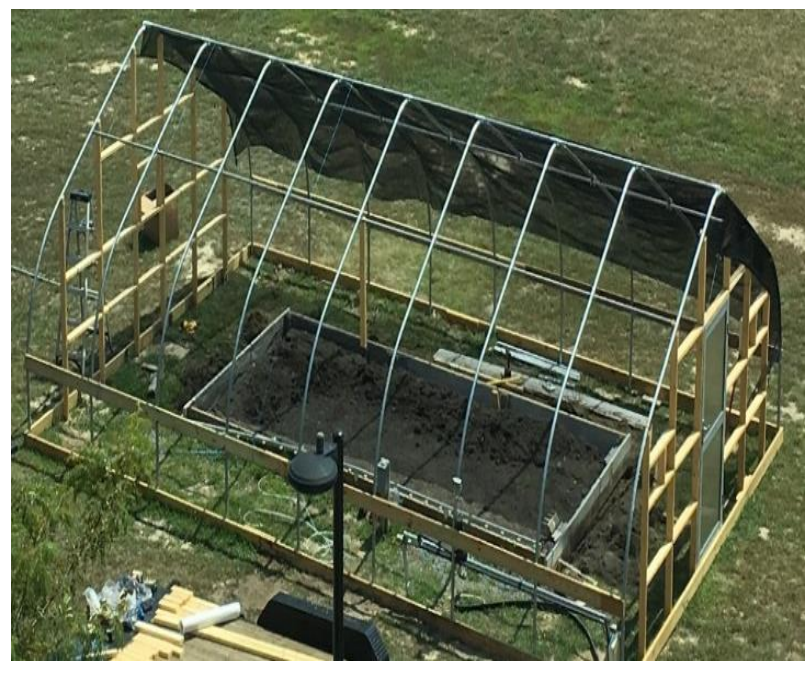

Figure 8: Farmbot Hoop House Frame

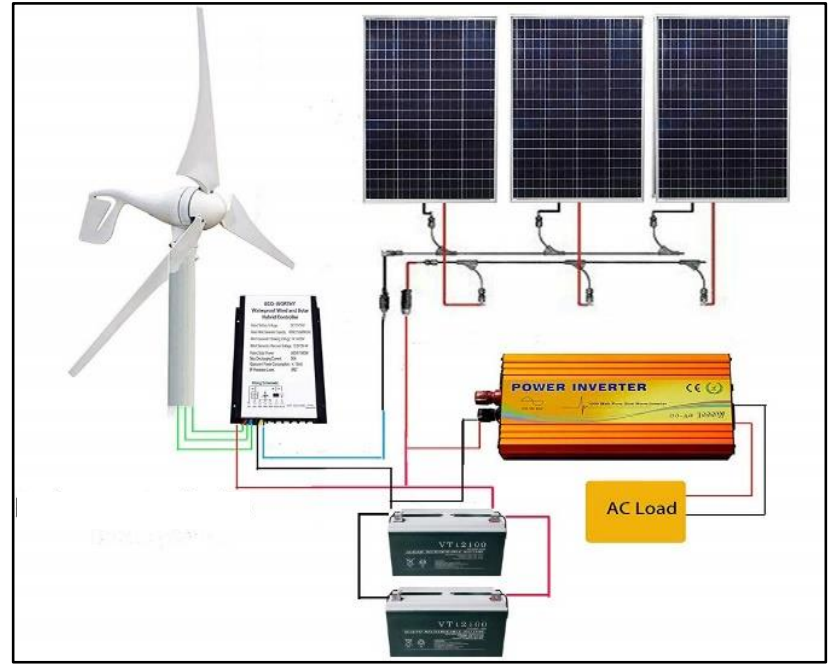

Figure 9: Wind and Solar Power Set-up

Over the last few months, the project team has completed the installation of the hoop house (Figure 8) to extend the growing season. Also, significant progress has been made with the installation of wind and solar power generation arrangement to power the FarmBot (Figure 9). The system has been installed and is generating power. Some wiring issues needs to be completed with the supervision of physical plant personnel (electrician) so that the FarmBot can be switched between regular power and the renewable power generated by the set-up. While the wind and solar set up should be capable of taking care of all the power needs of the FarmBot operations, the ability to switch to regular power will allow the machine to be operational even during the maintenancerelated downtime of the renewable energy generation system. The basic plan for the rainwater harvesting system for the FarmBot has been developed and most of the necessary components have been acquired. The preliminary installation efforts are underway. 


\subsection{Immediate and Future Plans}

As mentioned earlier the immediate plans (Spring 2020) for the project is to conduct a field trial with growing radish and arugula in the FarmBot bed. Figure 10 illustrates the details of the experimental layout. The FarmBot bed will accommodate the design that includes four randomized irrigation levels for each vegetable including a control (no irrigation) that has been arranged in three blocks for each. The vegetables have been chosen for quick turnaround time. The harvest data will be collected for each area indicated in the Figure and a randomized block design one way ANOVA analysis will be conducted followed by a mean separation test (Tukey) using suitable statistical software. The output data (dependent variable) will be estimated from the harvested biomass as well as images of the plants acquired by the camera attached to the FarmBot at suitable growth stages.

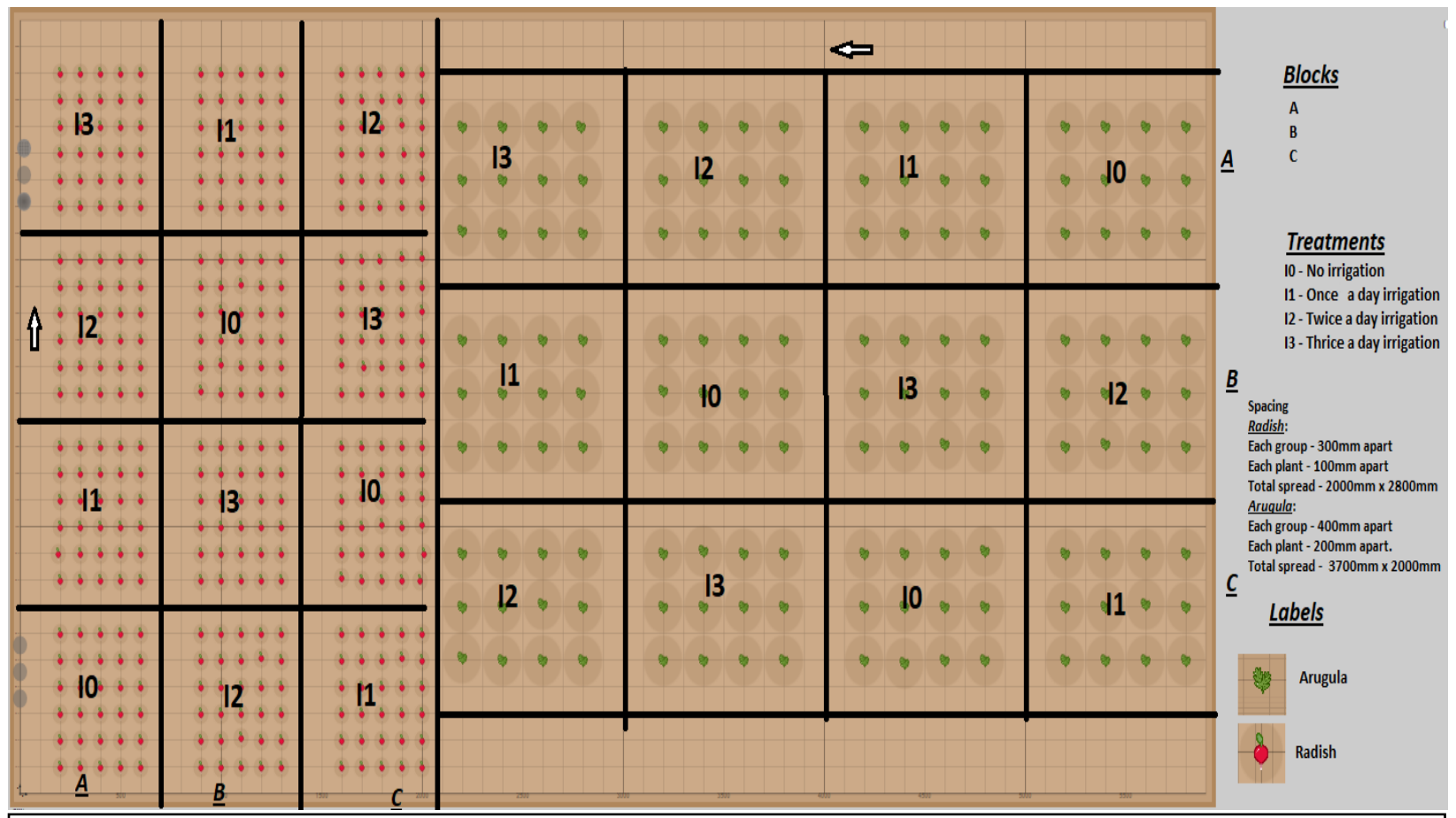

Figure 10: Experimental Design for Radish and Arugula ( RCB One way ANOVA)

Future plans also include using data from several similar trials to predict harvest biomass using artificial intelligence techniques by training an appropriate convolutional neural network (CNN) [9] using descriptive statistics data derived from overhead images of the plants at appropriate growth stages. The project team also plans to conduct trials with two factors by varying both irrigation and nitrogen levels to study the impact on the growth of specialty vegetables and other medicinal plants in the future.

All field trials will utilize the autonomous planting, weeding, sensing, watering and time-lapse photography capabilities of the machine. 
The FarmBot can be used in open yards, inside greenhouses, as well as indoor areas with grow light attachments. A FarmBot system with a smaller bed will be installed inside a suitable room in the Food Science and Technology building on campus in the next phase of the project. The project team will identify suitable grow lights and work out efficient operational logistics for an indoor set-up consistent with the green and sustainable emphasis of the overall scope of the proposed project. Experiments are being planned to study the impact of light levels, irrigation levels, as well as nutrient levels with the indoor setup.

Please note that COVID-19 related restrictions on campus have impacted the spring 2020 plans for the project as outlined. At the time of revising this document ( $2^{\text {nd }}$ week of April 2020) access to campus for students as well as faculty are severely regulated following social distancing protocols. The project team will work out appropriate plans to execute the project goals as regulations get relaxed and normal campus operations are resumed.

\subsection{Project Impact and Learning Outcomes}

The overarching goal of the FarmBot project is not only to conduct field trials that are at the nexus of food, water, and energy but also to provide an experiential learning and research platform that promotes learning, discovery, and engagement aligned with the land grant mission of the campus for a diverse multidisciplinary team of UMES students. The project engages the student team through all four stages of Kolb's experiential learning paradigm [10]. Active experimentation is informed by abstract concepts gathered in classrooms, as well as through concrete experiences and reflective observations during project meetings and task execution.

The undergraduate students involved in the project have realized the value of out of classroom hands-on experiences in broadening outlook, enhancing their resumes, and, enriching their overall educational experience at the university. For the graduate students in Food Science and Technology involved in the effort, the project has provided a platform for generating research ideas as well as to execute field experiments efficiently.

For the engineering students who are involved in the project team, the ABET outcomes that are mentioned in the classroom have taken on a new meaning. Although no effort is made to assess the ABET outcomes [11] in the context of the project the students readily acknowledge that they have learned to apply knowledge of science, engineering and mathematics in executing the project goals. The students have also realized the need to cultivate the ability to work in multidisciplinary teams in accomplishing assigned tasks. Also, discussions about food, water, and energy security that have taken place during project meetings among the faculty and students working in the project have made the students realize the global impact of engineering solutions that take into account environmental, social, economic, as well as other considerations. 


\subsection{Acknowledgment}

The authors gratefully acknowledge the support of university staff and administration to execute the project efforts. Also, efforts of all the undergraduate students who have attended project meetings and contributed to the project are acknowledged gratefully. Project leaders and the students also appreciate the funding support from the Maryland Space Grant Consortium (MDSGC) and the National Institute of Food and Agriculture (NIFA).

\section{References}

[1]. A.Nagchaudhuri, T.W. Ford, and C. Hartman, "Overview of Remote Sensing Efforts at University of Maryland Eastern Shore." Proceedings of the ASME 2019 International Design Engineering Technical Conferences and Computers and Information in Engineering Conference. Volume 9: 15th IEEE/ASME International Conference on Mechatronic and Embedded Systems and Applications. Anaheim, USA. August 18-21, 2019. V009T12A027. ASME. https://doi.org/10.1115/DETC2019-98457

[2]. A.Nagchaudhuri, M.Mitra, C.Hartman, T.W.Ford, T., and J.Pandya, "Mobile Robotic Platforms to Support Smart Farming Efforts at UMES", Proceedings of 14th IEEE/ASME Mechatronics and Embedded Systems Applications, July 2-4, 2018, Oulu, https://ieeexplore.ieee.org/document/8449182

[3]. B.Schoenfeld, "How the Farm-to-Table Movement Is Helping Grow the Economy". Entrepreneur, September 2011 Retrieved January 2020

[4]. S.Mekhilef, S.Z.Faramarzi , R.Saidur , Z.Salam (2013) The application of solar technologies for sustainable development of agricultural sector Renewable and Sustainable Energy Reviews, 18, pp. 583-594.

[5]. R.L.Aronson, Farmbot: Humanity's Open-Source Automated Precision Farming Machine. Tech. Farmbot, 19 Sept. 2013. https://farm.bot/blogs/news/the-farmbot-whitepaper

[6] S. Singh, M.Singh, and S.C Kaushik. (2016) A review of optimization techniques for sizing of solar-wind hybrid energy systems. International Journal of Green Energy 13:15, pages 1564-1578.

[7]. R.Dabhi, N. Bhatt and B. Pandit, 2013. Super absorbent polymers - An Innovative water-saving technique for optimizing crop yield. International Journal of Innovative Research in Science, Engineering, and Technology. 2(10):5333-53401.

[8]. N.Fidelia, and B.Chris, Environmentally friendly superabsorbent polymers for water conservation in agricultural lands. Journal of Soil Science Environmental Management., 2011, 2(7), 206-21

[9]. Y.Adhitya, S.W.Prakosa, M.Köppen, J.S.Leu. (2019) Convolutional Neural Network Application in Smart Farming. In: Berry M., Yap B., Mohamed A., Köppen M. (eds) Soft Computing in Data Science. SCDS 2019. Communications in Computer and Information Science, vol 1100. Springer, Singapore

[10]. Kolb, D. A. (1984). Experiential learning: Experience as the source of learning and development (Vol. 1). Englewood Cliffs, NJ: Prentice-Hall.

[11]. ABET EAC Outcomes [Online] : Available : https://www.abet.org/accreditation/accreditation-criteria/criteriafor-accrediting- engineering-programs-2019-2020/\#GC3 Accessed:[April 9,2020] 